Introduction The effect of this deletion has an impact on development of various organs and glands. 16 p13.3 microdeletion is a rare genetic condition with a variable phenotype spectrum. RBFOX1 [RNA binding protein] located on chromosome $16 \mathrm{p} 13.3$, is one of the three members in Fox gene family encoding splicing factors. We would like to present this case report to elucidate the association of this alteration with hypothyroidism and epileptic seizures as only one case of this association has been reported in the literature.

Case report An 8 year old boy presented with recurrent afebrile, unproked seizures and behaviour difficulties. EEG was supportive for multifocal epilepsy. The MRI brain scan was normal. Blood results showed persistently high TSH levels and low free T4. Subsequent workup showed a high TPO level and the endocrine team made a diagnosis of autoimmune hypothyroidism. Genetics investigations showed that the patient had a 16 p13.3 microdeletion. A similar genotype was noted in the mother though she was phenotypically normal.

Discussion RBFOX1 is an important splicing factor identified as a binding protein of ATXN2, suggesting a role in neurologic function. It is highly expressed in the brain, skeletal muscle, heart, other organs and glands. Any alteration with this specific locus is associated with variable phenotypes affecting various body systems. These include mental retardation, epilepsy, mental health disorders, congenital heart defects, obesity and diabetes along with other endocrine problems. The introduction of array comparative genomic hybridization $(\mathrm{CGH})$ has provided the ability to map DNA copy number variations (CNVs) genome with high resolution. Only one patient with this $\mathrm{CNV}$ has been reported to have congenital hypothyroidism and associated epilepsy. In our patient though the CGH array has helped in mapping out the genetic defect howver its role in hypothyroidism is yet to be established.

Conclusion Patients presenting with multiple system involvement, especially involving the endocrine system with associated developmental delay and neurobehavioral and neuropsychiatric problems should undergo CGH array analysis as part of their endocrine assessment. Moreover endocrinologists need to be aware of this copy number variation as it may have a huge impact on future pregnancies of the patient.

\section{G317(P) ABSTRACT WITHDRAWN}

\section{G318(P) SCN1A-RELATED EPILEPSY: THREE DIFFERENT CASES AND A LITERATURE OVERVIEW}

${ }^{1} \mathrm{~T}$ Ratnaike, ${ }^{1} \mathrm{~K}$ Moodley, ${ }^{1} \mathrm{~A}$ Saraswatula, ${ }^{2} \mathrm{G}$ Ambegaonkar. ${ }^{1}$ Paediatric Department, West Suffolk Hospital, Bury St Edmunds, UK; '²Department of Neurology, Addenbrookes Hospital, Cambridge, UK

\subsection{6/archdischild-2018-rcpch.308}

Among epilepsies caused by a single gene mutation, the sodium channel neuronal type $1 \alpha$ subunit (SCN1A) gene, encoding the voltage-gated sodium channel $\mathrm{Na}_{\mathrm{v}} 1.1$, is the most frequently associated with epilepsy (Brunklaus et al. 2014).
The mutations cause a spectrum of disease including genetic epilepsy with febrile seizures plus (GEFS+), Dravet syndrome or severe myoclonic epilepsy of infancy (SMEI), and borderline severe myoclonic epilepsy of infancy (SMEIB). We describe three children diagnosed with SCN1A-associated atypical epilepsy phenotypes whose treatment continues to be challenging. We report 3 children less than 12 months presenting with multiple pharmacoresistant seizure types proving diagnostic and clinical challenge. All present with a heterozygous sequence change identified in SCNIA gene.

As per McDonald and colleagues (2017), carbamazepine, oxcarbazepine, and lamotrigine may worsen focal seizures associated with SCN1A mutation, or have other adverse effects. Sodium valproate, stiripentol, and clobazam are thought to have the best impact on management of focal seizures such as those seen in Dravet syndrome. Carbamezapine was also seen to exacerbate seizures in GEFS +epilepsy positive for SCN1A mutation (Shi et al. 2016).

The establishment of the SCN1A variant database may aid further therapy development targeted to specific genetic mutations (Claes et al. 2009).Cannabidiol, ketogenic diet, fenfluramine therapies are novel therapies used in the treatment of this highly pharmaco resistant form of epilepsy.

SCN1A-related epilepsy consists of a spectrum of disease that is still difficult to manage due to the different seizure types present and treatment resistance. This is certainly true of our three cases, all three are female children who presented in infancy and have suffered from an evolving clinical course. They are all being treated with stiripentol, however, have not achieved seizure control, and it will be vital to keep updated with ongoing research into newer therapies.

\section{G319(P) OUTCOMES FOLLOWING FEBRILE CONVULSIONS: A RETROSPECTIVE COHORT STUDY}

${ }^{1} \mathrm{~L}$ Chatterton Dickson, ${ }^{1,2} \mathrm{~J}$ Shetty. 'Edinburgh Medical School, University of Edinburgh, Edinburgh, UK; ${ }^{2}$ Child Life and Health, Royal Hospital for Sick Children, Edinburgh, UK

\subsection{6/archdischild-2018-rcpch.309}

Febrile convulsions (FC) are seizures in children under five associated with fever. They are reported in $2 \%-5 \%$ of children with good prognosis. However, the risk of epilepsy may be increased. This study reported the first Scottish cohort and observed current clinical practice, outcome and associations with socioeconomic status. A FC database was created using routinely collected clinical data of all children attending $\mathrm{A}$ and E. A Scottish prevalence of 390/100,000 children/year was elucidated with $2.6 \%$ of children experiencing FCs by their fifth birthday. Children from deprived areas were overrepresented. It is established that only very young children or children with complex convulsions should be admitted and further investigations are usually unnecessary. However, $72.6 \%$ of children were admitted and $10.4 \%$ received an EEG which is inappropriately high. Epilepsy was diagnosed in $2.6 \%$ of children, an increase from the $1 \%$ population risk. However, many of these children had other abnormalities. Only $1.5 \%$ of otherwise healthy children developed epilepsy. Overall, the danger of FCs should not be overstated as they only slightly increase the risk of epilepsy in an otherwise healthy child. This should be explained to quell parental anxieties and reduce unnecessary admissions and investigations. 